\title{
Fixed point properties for semigroups of non-expansive mappings in conjugate Banach spaces
}

\author{
Khadime Salame
}

\begin{abstract}
In this paper we study common fixed point properties of non-linear actions of semi-topological semigroups on non-void weak* compact convex sets in dual Banach spaces. Among other things, we derive from our main result Theorem 1, the existence of a common fixed point property for semigroups of non-expansive mappings acting on non-empty weakly compact convex sets, generalizing a result of Hsu [13], Mitchell [25].
\end{abstract}

\section{Introduction}

Let $K$ be a non-empty subset of Banach space $E$. We say that a mapping $T: K \rightarrow K$ is non-expansive if

$$
\|T(x)-T(y)\| \leq\|x-y\|, \text { for all } x, y \in K .
$$

A bounded closed convex subset $C \subset E$ is said to have normal structure, if for all closed convex subset $W$ of $C$ such that $\delta(W)>0$ (i.e., with positive diameter), there is $x \in W$ such that:

$$
\sup _{y \in W} q(x-y)<\sup _{y, z \in W} q(y-z)
$$

Received by the editors in May 2016.

Communicated by F. Bastin.

2010 Mathematics Subject Classification : 47H10, 47H20.

Key words and phrases : amenable semigroups, asymptotic normal structure, fixed point properties, left reversible semigroups, non-expansive mappings, normal structure. 
The notion of normal structure was introduced by Brodskii and Milman (see [4]), when they studied fixed points of isometries. During the 60's, DeMarr in [7] showed that norm compact convex subsets of Banach spaces possess normal structure. This result played a crucial key on DeMarr's proof on the existence of a common fixed point for commuting families of non-expansive mappings on non-empty compact convex sets in Banach spaces. Takahashi in [29], extended DeMarr's result by considering left amenable discrete semigroups of non-expansive mappings. Mitchell in [25], improved Takahashi's result by showing that it still holds even for left reversible semigroups of non-expansive mappings (note that a left amenable discrete semigroup is always left reversible). A generalization of Mitchell's result to left reversible semi-topological semigroups was established by Lim in [22] to separated locally convex spaces, and by Lau and Holmes in [12] with some continuity assumption. A related result was proved by Hsu [13], where he showed that every weakly continuous non-expansive action of a left reversible discrete semigroup on a non-empty weakly compact convex subset of a Banach space possesses a common fixed point. When we deal with weak topologies, one cannot in general avoid the use of normal structure for non-expansive mappings without additional assumption. Indeed, it was proved by Alspach [1] in 1981 that there is a non-expansive mapping on a non-void weakly compact convex set into itself that is fixed point free. In 1965, Kirk [16] proved a surprising result showing that a non-expansive self-map on a weakly compact convex set with normal structure has a fixed point. Hsu's result shows that if we restrict to the subclass of weakly continuous non-expansive mappings, then normal structure can be avoided. A modification of Kirk's proof shows that his result can be extended to conjugate Banach spaces with the weak* topology. Karlovitz [15] proved that in $\ell^{1}$, any non-expansive mapping on a bounded weak ${ }^{*}$ closed convex subset has a fixed point. Lim [23] extended this result to left reversible semi-topological semigroups of non-expansive mappings.

A semigroup $S$ is said to be a semi-topological semigroup, if it has a Hausdorff topology such that, for all $s \in S$, the following mappings : $t \mapsto$ s.t and $t \mapsto t . s$ from $S$ into itself, are continuous. A non-expansive action of $S$ on $K$, is a mapping $\mathfrak{S}: S \times K \rightarrow K$ such that for all $s, t \in S$ and for all $x \in K$ we have :

$$
\mathfrak{S}(s t, x)=\mathfrak{S}(s, \mathfrak{S}(t, x))
$$

and $x \mapsto \mathfrak{S}(s, x): K \rightarrow K$ is non-expansive. For short, we shall denote the value $\mathfrak{S}(s, x)$ of the mapping $\mathfrak{S}$ at the point $(s, x)$, by the symbol "s.x" or sometimes by "रि( $(x)$ ".

Given an action of $S$ on $K$, an element $x \in K$ is said to be a common fixed point for $S$ if it is subject to the condition $\widehat{s}(x)=x$ for all $s \in S$. The collection of all such $x$ in $K$ is called the fixed point set of $S$ and denoted by $F(S)$.

When $S$ is semi-topological, let $\mathfrak{S}: S \times X \rightarrow X$ be a representation of $S$ on a topological space $X$. $\mathfrak{S}$ is said to be jointly continuous, if it is continuous whenever $S \times X$ is given the product topology. A subset $C$ of $X$ is called $S$-invariant, if s.C $:=\mathfrak{S}(s, C) \subset C$ for all $s \in S$. 
Given a semi-topological semigroup $S$, we shall denote by $C_{b}(S)$ the Banach algebra of all bounded continuous real-valued functions on $S$, with the sup norm. Let $\Phi$ be a closed subspace of $C_{b}(S)$. We say that $\Phi$ is left translation invariant, if it has the following property :

$$
\text { For all } f \in \Phi \text { and } s \in S \text {, we have } \ell_{s} f \in \Phi
$$

where the operator $\ell_{s}: C_{b}(S) \rightarrow C_{b}(S)$ is defined by the formula $\ell_{s} f(t)=f(s t)$, for all $t \in S$. The element " $\ell_{s} f$ " is called the left translate of $f$ by $s$. Analogously, we define the right translation operator $r_{s}$ and the right translate $r_{s} f$ of $f$ by $r_{s} f(t):=$ $f(t s)$. If $\Phi$ is a left translation invariant subspace of $C_{b}(S)$ containing the constant functions on $S$, a member $m$ of $\Phi^{*}$ (topological dual of $\Phi$ ) is called a mean on $\Phi$, if $m(e)=1=\|m\|$.

A mean $m$ on $\Phi$ is called left invariant if it satisfies the following equation :

$$
m\left(\ell_{s} f\right)=m(f) \text { for all } f \in \Phi \text { and for all } s \in S \text {. }
$$

We say that the subspace $\Phi$ is left amenable, if it possesses a left invariant mean. For short, we write " $\Phi$ has a LIM". A multiplicative mean on $\Phi$, is a mean $m$ such that

$$
m(f . g)=m(f) \cdot m(g), \text { for all } f, g \in \Phi
$$

Let LUC $(S)$ be the subspace of $C_{b}(S)$ of those functions $f$ such that the mapping $t \mapsto \ell_{t} f: S \rightarrow C_{b}(S)$ is continuous when $C_{b}(S)$ is given the sup norm topology. The elements of $\operatorname{LUC}(S)$ are called left uniformly continuous functions on $S$. It is well-known that $\mathrm{LUC}(S)$ is a translation invariant (i.e. left and right invariant) closed sub-algebra of $C_{b}(S)$ and contains constants functions (see [2] or [24]). The Banach algebra LUC $(S)$ was introduced jointly by Mitchell and Itzkowitz (see [14]). By misuse of language, we shall say that a semi-topological semigroup $S$ is left amenable, if $\mathrm{LUC}(S)$ is.

Example 1. When $S$ is a topological group, then $\operatorname{LUC}(S)$ is the set of all uniformly continuous functions on $S$ with respect to the right uniformity of $S$ i.e., $f \in \operatorname{LUC}(S) \Leftrightarrow \forall \epsilon>0, \exists U$ neighborhood of the identity of $S$ such that $s^{-1} t \in U \Rightarrow|f(s)-f(t)| \leq \epsilon$. See [11] for more details.

The following properties are well-known (see [2]):

- If $S$ is a discrete semigroup, then $\operatorname{LUC}(S)=C_{b}(S)=\ell^{\infty}(S)$.

- If $S$ is a compact topological semigroup (i.e., the operation of $S$ is jointly continuous), then $\operatorname{LUC}(S)=C_{b}(S)$.

A semi-topological semigroup $S$ is called left reversible, if any two closed right ideals intersect i.e.,

$$
\overline{a . S} \cap \overline{b . S} \neq \varnothing \text {, for all } a, b \in S \text {. }
$$


When $S$ is discrete, then $S$ left amenable implies $S$ left reversible. Note that the converse is not true in general e.g., just consider a non-amenable group (free group on two generators see [5] and [6]). If we consider the topological case, a left amenable semi-topological semigroup need not be left reversible. Indeed, Hewitt[10] has constructed a regular Hausdorff topological space $S$ such that the only continuous real-valued functions on it are constant functions; in [9], Granirer defined a semi-topological semigroup structure on $S$ by letting $a . b=a$ for all $a, b \in S$. Then it is easy to see that for all $a \in S$ the point mass $f \mapsto \delta_{a}(f)=f(a)$ defines a left invariant mean on $C_{b}(S)$. However, $S$ is not left reversible since, if $a \neq b,(\{a\}=\overline{a . S}) \cap(\overline{b . S}=\{b\})=\varnothing$. See also [12].

\section{Main Results}

In this section, we shall present our main results. We first give the following definitions which will be used in the sequel. Let $S$ be a semi-topological semigroup.

- $S$ is said to be sequentially left amenable, if there is a left invariant mean $m \in \operatorname{LUC}(S)^{*}$ and a sequence $\left(m_{n}\right)_{n \in \mathbb{N}}$ of finite means such that $m=$ weak $^{*}$ $\lim _{n} m_{n}$. In this case, we shall write "S is seq-LA".

The class Seq-LA of all sequentially left amenable semi-topological semigroups was introduced by the author (see [28]). It contains all countable left amenable discrete semigroups (see [28, theorem 2.3]), all compact metrizable left amenable semi-topological semigroups.

- $S$ is said to be $\sigma$-left amenable, if there is a family $\left(S_{\gamma}\right)_{\gamma \in \Gamma}$ of sub-semi-topological semigroups subject to the following conditions:

1. $S=\cup_{\gamma} S_{\gamma}$

2. For all $\gamma, \gamma^{\prime} \in \Gamma$, there is $\gamma^{\prime \prime} \in \Gamma$ such that $S_{\gamma} \cup S_{\gamma^{\prime}} \subset S_{\gamma^{\prime \prime}}$;

3. For all $\gamma \in \Gamma, S_{\gamma}$ is separable;

4. For all $\gamma \in \Gamma, \operatorname{LUC}\left(S_{\gamma}\right)$ has a LIM.

The class $\Gamma$-LA of all $\sigma$-left amenable semi-topological semigroups contains trivially all separable left amenable semi-topological semigroups and all amenable locally compact topological groups (due to the fact that each closed subgroup is amenable).

Example 1. Discrete left amenable semigroups are in $\Gamma$-LA. Indeed, given a discrete left amenable discrete semigroup $S$, we know that each countable subsemigroup of $S$ is contained in some countable left amenable one (see [8]). Define

$$
\mathcal{S}:=\{Z \subset S ; Z \text { is a left amenable countable sub-semigroup }\} .
$$

Note that $\mathcal{S}$ is non-void because if we fix $s \in S, \mathcal{S}$ contains the commutative semigroup $\langle s\rangle$ generated by $s$ which is countable an amenable. We order $\mathcal{S}$ by letting $Z \leq Z^{\prime} \Leftrightarrow Z \subset Z^{\prime}$. Then it is clear that $S=\bigcup_{Z \in \mathcal{S}} Z$ and given $Z, Z^{\prime} \in \mathcal{S}$, there is 
$Z^{\prime \prime} \in \mathcal{S}$ such that $Z \cup Z^{\prime} \subset Z^{\prime \prime}$. Because $Z$ and $Z^{\prime}$ being countable, it follows that $\left\langle Z \cup Z^{\prime}\right\rangle$ is countable too, and we choose $Z^{\prime \prime} \in \mathcal{S}$ such that $Z^{\prime \prime} \supset\left\langle Z \cup Z^{\prime}\right\rangle$ using $[8$, theorem E1].

- $S$ is said to be strongly left reversible, if there is a family $\left(S_{\gamma}\right)_{\gamma \in \Gamma}$ of countable left reversible sub-semigroups of $S$ satisfying the conditions 1 and 2 of the previous definition.

The class of all strongly left reversible semi-topological semigroups was introduced in [19] by Lau and Zhang. It includes all discrete left reversible semigroups (see [13]), all separable left reversible semi-topological semigroups and all metrizable left reversible semi-topological semigroups see [19].

\subsection{Common fixed point properties in dual spaces}

Given a Banach space $E$, let $B_{E^{* *}}$ denote the unit closed ball of the second dual $E^{* *}$; and let $\operatorname{Ext}\left(B_{E^{* *}}\right)$ be the set of all extreme points of $B_{E^{* *}}$ (which is of course nonvoid by virtue of the Krein-Milman theorem). Consider on the dual $E^{*}$ the locally convex topology $\tau$ defined by the family of semi-norms $Q:=\left\{p_{e}\right.$; $\left.e \in \operatorname{Ext}\left(B_{E^{* *}}\right)\right\}$ where, $p_{e}(f)=|e(f)|$; then using the Krein-Milman theorem, it is easy to see that $\tau$ is separated. On the other hand, by construction $\tau$ is weaker than the weak topology $\sigma\left(E^{*}, E^{* *}\right)$.

In this section, the notations $\bar{A}^{\tau}$ and $\overline{c o}^{\tau}(A)$ will stand respectively for the closure and closed convex hull of a subset $A \subset E^{*}$ with respect to a locally convex topology $\tau$ on $E^{*}$.

Theorem 1. Let $S$ be a semi-topological semigroup. Assume that it satisfies either one of the following conditions :

1. $S$ is $\sigma$-LA;

2. $S$ is seq-LA;

3. $S$ is strongly left reversible.

Then $S$ possesses the following fixed point property :

$\left(F_{\tau}^{*}\right)$ : Whenever $S \times K \rightarrow K$ is a weak ${ }^{*}$ jointly continuous non-expansive action on a non-empty weak ${ }^{*}$ compact convex subset $K$ of a dual $E^{*}$ of a Banach space $E$, such that for all non-void weak* closed and $S$-invariant subset $B$ of $K$ with the property $s . B=B$ for all $s \in S$, there is $x \in B$ whose orbit $\mathcal{O}_{x}$ is relatively countably $\tau$-compact, then there is in $K$ a common fixed point for $S$.

In order to prove this result, the following lemmas are needed :

Lemma 1. Whenever $S$ defines a jointly continuous action on a compact topological space $M$, then for all $x \in M$ and $f \in C(M)$ the mapping $\theta_{x}^{f}: S \rightarrow \mathbb{R}, s \mapsto$ $f(s . x)$ lies in $\operatorname{LUC}(S)$.

Proof. See [25, proof of theorem 1]. 
Lemma 2. For each non-void weak* compact and $S$-invariant subset $K^{*}$ of $K$, there is a minimal non-empty weak ${ }^{*}$ compact set $\Omega^{*} \subset K^{*}$ such that $s . \Omega^{*}=\Omega^{*}$, for all $s \in S$.

Proof. See [28, lemma 2.12].

Lemma 3. Let $\Omega^{*}$ be as in the previous lemma. Then the following facts hold :

1. For all $x \in \Omega^{*}$, the orbit $\mathcal{O}_{x}:=\{s . x ; s \in S\}$ of $x$, is weak ${ }^{*}$ dense in $\Omega^{*}$.

2. $\Omega^{*}$ is $\sigma\left(E^{*}, E^{* *}\right)$-compact.

For the proof of this lemma, we shall need the following well-known characterization of weak relative compactness in Banach space theory (see [27]) :

Lemma 3.1. Let $B$ be a Banach space and $C$ be a non-empty bounded subset of $B$. $C$ is relatively weakly compact if and only if, for all sequence $\left(x_{n}\right)_{n}$ in $C$, there is a sequence $\left(y_{n}\right)_{n} \in E$ such that $y_{n} \in \operatorname{co}\left(x_{i} ; i \geq n\right)$ for all $n$ that is weakly convergent.

Proof of Lemma 3. For part 1, clearly orbits are S-invariant; and since for all $s$ in $S$, the mapping $x \mapsto$ s. $x$ is weak ${ }^{*}$-weak ${ }^{*}$ continuous (due to the continuity of the action) then weak ${ }^{*}$ closures of orbits are also $S$-invariant. Hence, by minimality, it follows that 1 holds. For part 2, from lemma 2 we have that $\Omega^{*}$ is non-empty with the property $s . \Omega^{*}=\Omega^{*}$ for all $s \in S$. Let us fix $x \in \Omega^{*}$ with relatively $\tau$-compact orbit; and let $\left(z_{n}\right)_{n}$ be a sequence in $\mathcal{O}_{x}$. Since the orbit $\mathcal{O}_{x}$ is bounded (as a subset of $K)$, then so is $\left(z_{n}\right)_{n}$; therefore, by [26, corollary 0.2$]$, there is a subsequence $\left(z_{n_{k}}\right)_{k}$ of $\left(z_{n}\right)_{n}$ such that:

$$
\bigcap_{k=1}^{\infty} \overline{c o}^{\tau}\left(z_{n_{i}} ; i \geq k\right) \subset \bigcap_{n=1}^{\infty} \overline{c o}\left(z_{i} ; i \geq n\right) .
$$

Define $F_{n}:={\overline{\left\{z_{n_{i}} ; i \geq n\right\}}}^{\tau}$ for all $n$. Then $\left\{F_{n} ; n \in \mathbb{N}\right\}$ is a decreasing sequence of $\tau$-closed non-empty subsets of the countably $\tau$-compact space $\overline{\mathcal{O}}_{x}^{\tau}$; therefore, $\bigcap_{n=1}^{\infty} F_{n} \neq \varnothing$; and this implies a fortiori, the existence of $\xi \in \bigcap_{n=1}^{\infty} \overline{c o}\left(z_{i} ; i \geq n\right)$. Thus it follows the existence of a sequence $\left(\xi_{n}\right)_{n} \in E^{*}$ such that $\xi_{n} \in \operatorname{co}\left(z_{i} ; i \geq n\right)$ for all $n$ and $\left\|\xi_{n}-\xi\right\| \rightarrow 0$. Hence by lemma 3, it follows that ${\overline{\mathcal{O}_{x}}}^{\text {wk }}$ is weakly compact; and therefore weakly* closed and together with the first part, we deduce that

$$
\Omega^{*}=\overline{\mathcal{O}}_{x}{ }^{w *}={\overline{\mathcal{O}_{x}}}^{w k} \text {. }
$$

Hence, $\Omega^{*}$ is a weakly compact space.

Lemma 4 . Let $\Omega^{*}$ be as in the previous lemma. If $S$ is a separable or a seq-LA semi-topological semigroup, then $\Omega^{*}$ is compact in the norm topology.

Proof. We first show that $\Omega^{*}$ is separable in the norm topology. Note that on $\Omega^{*}$ weak and weak* topologies agree (see lemma 3 ).

- If $S$ is seq-LA, let us pick $x \in \Omega^{*}$ and consider $\left(m_{n}\right)_{n}$ be a sequence of finite means converging pointwise to a $\operatorname{LIM} m$ on $\operatorname{LUC}(S)$. Define $\psi: C\left(\Omega^{*}\right) \rightarrow \mathbb{R}$ by 
$\psi(f):=m\left(f_{x}\right)$, with $f_{x}(s)=f(s . x)$ ( $\psi$ is well-defined by lemma 1 ). As readily checked, $\psi$ is a non-zero non-negative linear functional. By the Riesz representation theorem, there is a regular Borel measure $\mu$ on $\Omega^{*}$ such that $\psi(f)=\int_{\Omega^{*}} f d \mu$ which is moreover a probability $\left(\mu\left(\Omega^{*}\right)=1\right)$. Let $\omega_{*}$ be the support of $\mu$. It is easy to see that $\omega_{*}$ is characterized by :

$$
x \in \omega_{*} \Leftrightarrow \forall V \in \mathscr{V}_{w k}(x), \mu\left(V \cap \omega_{*}\right)>0 .
$$

Where $\mathscr{V}_{w k}(x)$ denotes the collection of all weak neighborhoods of $x$ in $E$. For all $n \in \mathbb{N}$, let $m_{n}=\sum_{i=1}^{\alpha_{n}} t_{i} \delta_{s_{i}^{n}}$. Then for $n$ fixed, $\psi_{i}^{n}: f \mapsto f\left(s_{i}^{n} \cdot x\right)$ is a non-zero multiplicative linear functional on $C\left(\Omega^{*}\right)$; therefore there is $x_{i}^{n} \in \Omega^{*}$ such that $\psi_{i}^{n}(f)=f\left(x_{i}^{n}\right)$. We claim that

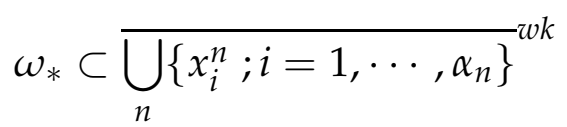

(the closure being taken with respect to the weak topology). Indeed, if $y$ is a point outside this (weakly) closed set, then by Urysohn's lemma, there is $f \in C\left(\Omega^{*}\right)$ such that $f \geq 0, f(y)=1$ and $f\left(x_{i}^{n}\right)=0$ for all $\mathrm{n}$ and $i=1, \cdots, \alpha_{n}$. Then $V:=$ $\{f>0\}$ is a neighborhood of $y$ (in the weak topology) and furthermore we have

$$
\begin{aligned}
\mu\left(V \cap \omega_{*}\right) \leq \int_{V \cap \Omega^{*}} f d \mu & \leq \int_{\Omega^{*}} f d \mu=\psi(f)=m\left(f_{x}\right) \\
& =\lim _{n} \sum_{i=1}^{\alpha_{n}} t_{i}^{n} \delta_{s_{j}^{n}}\left(f_{x}\right)=\lim _{n} \sum_{i=1}^{n} t_{i}^{n} \psi_{i}^{n}(f) \\
& =\lim _{n} \sum_{i=1}^{n} t_{i}^{n} f\left(x_{i}^{n}\right)=0
\end{aligned}
$$

Therefore $y \notin \omega_{*}$, since $\mu\left(V \cap \omega_{*}=0\right.$; which proves our claim. Hence $\omega_{*}$ as a subset of a weakly separable set (therefore norm separable) is a fortiori norm separable. On the other hand, by [28], $\omega_{*}$ satisfies $s . \omega_{*}=\omega_{*}$ for all $s \in S$. Thus by minimality, $\Omega^{*}=\omega_{*}$ is norm separable.

- Now we assume that $S$ is separable. Let $D \subset S$ be a dense subset and fix $x_{0} \in \Omega^{*}$. We shall show that the countable set $\left\{d . x_{0} ; d \in D\right\}$ is dense in $\Omega^{*}$ in the weak topology. Let $y \in \Omega^{*}$ and $V \in \mathscr{V}^{*}(0)$ (fundamental system of convex neighborhoods of " 0 " in the weak* topology). We choose $s \in S$ such that s. $x_{o} \in y+\frac{1}{2} V$. Then by continuity of $z \mapsto z . x_{0}$, there is a neighborhood $U_{s}$ of $s$ in $S$ such that $z \in U_{s} \Rightarrow z . x_{o} \in s . x_{o}+\frac{1}{2} V$. Now let us fix $d \in D \cap U_{s}$. Then,

$$
\text { d. } x_{o} \in \text { s. } x_{o}+\frac{1}{2} V \subset y+\frac{1}{2} V+\frac{1}{2} V=y+V .
$$

Therefore we have :

$$
(y+V) \cap\left\{d . x_{o} ; d \in D\right\} \neq \varnothing \text { for all } V \in \mathscr{V}^{*}(0) .
$$

Thus, $y \in{\overline{\left\{d . x_{o} ; d \in D\right\}}}^{w k^{*}}$. Since $y$ is arbitrary, it follows that $\Omega^{*}=$ ${\overline{\left\{d . x_{0} ; d \in D\right\}}}^{\text {wk* }}$ is weak* separable. But, we know from lemma 3 that $\Omega^{*}$ is 
weakly compact. So weak and weak ${ }^{*}$ topologies must coincide; and this fact implies the weak separability of $\Omega^{*}$. Next, we justify that $\Omega^{*}$ is separable in the norm topology. Let $M:=\overline{\operatorname{span}}\left(d . x_{0} ; d \in D\right.$ ) (norm closed linear manifold generated by the $d . x_{o}{ }^{\prime}$ s, $d \in D$ ). The subset $\mathcal{D}:=\operatorname{span}_{\mathrm{Q}}\left(d . x_{o} ; d \in D\right.$ ) (linear manifold generated over $\mathbb{Q}$ ) is clearly countable and it is norm dense in $M$. Therefore $M$ is norm separable and a fortiori $\Omega^{*}$ (as a subspace of a separable metric space).

The second part of the proof is devoted to showing the norm compactness of $\Omega^{*}$. We follow an argument of Hsu [13] or in [20] for non-expansive mappings in locally convex spaces. Note that as $\Omega^{*}$ is norm closed, showing its compactness is equivalent to proving that it is totally bounded in the norm topology. So Let $\epsilon>0$ fixed. From the norm separability showed earlier, let $\Omega^{*}=\bar{\zeta}$ (norm closure of $\zeta$; a countable subset of $\left.\Omega^{*}\right)$. Then $\Omega^{*} \subset \bigcup_{\sigma \in \varsigma} B\left[\sigma, \frac{\epsilon}{2}\right]$. Since each closed ball $B\left[\sigma, \frac{\epsilon}{2}\right]$ is norm closed and convex, it is weakly closed. So $\left\{B\left[\sigma, \frac{\epsilon}{2}\right] \cap \Omega^{*}\right.$; $\sigma \in \varsigma\}$ is a countable weakly closed covering of $\Omega^{*}$. But $\Omega^{*}$ being weakly compact, it is a Baire space. Therefore there is $\tilde{\sigma} \in \mathcal{S}$ such that $B\left[\tilde{\sigma}, \frac{\epsilon}{2}\right] \cap \Omega^{*}$ has non-void interior in the relative weak topology. So let $x_{\epsilon} \in \Omega^{*}$ and $V_{\epsilon}$ be a weak neighborhood of the origin such that $\left(x_{\epsilon}+V_{\epsilon}\right) \cap \Omega^{*} \subset B\left[\tilde{\sigma}, \frac{\epsilon}{2}\right] \cap \Omega^{*}$. Then $\left(x_{\epsilon}+V_{\epsilon}\right) \cap \Omega^{*} \subset B\left[x_{\epsilon}, \epsilon\right]$. Indeed, if $z \in\left(x_{\epsilon}+V_{\epsilon}\right) \cap \Omega^{*}$ then, $z-x_{\epsilon} \in V_{\epsilon}$. Thus $\left\|x_{\epsilon}-z\right\| \leq\left\|x_{\epsilon}-\tilde{\sigma}\right\|+\|\tilde{\sigma}-z\| \leq \epsilon$. Now choose a weak neighborhood $V_{\epsilon}^{\prime}$ of "0" such that $V_{\epsilon}^{\prime}+V_{\epsilon}^{\prime} \subset V_{\epsilon}$. Then let $\delta_{\epsilon}>0$ such that $B\left[0, \delta_{\epsilon}\right] \subset V_{\epsilon}^{\prime}$ (this can be done because the norm topology is finer than the weak topology). Then we have $\Omega^{*} \subset \bigcup_{x \in \sigma} B\left[x, \delta_{\epsilon}\right]$. As $\sigma\left(E^{*}, E^{* *}\right)=\sigma\left(E^{*}, E\right)$ on $\Omega^{*}$, then orbits are also weakly dense in $\Omega^{*}$. Since $\varsigma$ is countable, let $\sigma:=\left\{\sigma_{i} ; i=1,2, \cdots\right\}$. Then by induction the following implications hold

For $\mathrm{n}=1, x_{\epsilon} \in{\overline{\mathcal{O}_{\sigma_{1}}}}^{w k} \Rightarrow \exists s_{1} \in S$ such that $\widehat{s_{1}}\left(\sigma_{1}\right)-x_{\epsilon} \in V_{\epsilon}^{\prime}$.

For $\mathrm{n}=2, x_{\epsilon} \in{\overline{\mathcal{O}_{s_{1} \cdot \sigma_{1}}}}^{w k} \Rightarrow \exists s_{2} \in S$ such that $\widehat{s_{1}}\left(\sigma_{2}\right)-x_{\epsilon} \in V_{\epsilon}^{\prime}$.

For $\mathrm{n}=3, x_{\epsilon} \in \overline{\mathcal{O}_{s_{2} s_{1} \cdot \sigma_{3}}} w k \Rightarrow \exists s_{3} \in S$ such that $\widehat{s_{3} s_{2} s_{1}}\left(\sigma_{3}\right)-x_{\epsilon} \in V_{\epsilon}^{\prime}$.

By induction for $\mathrm{n}=\mathrm{p}$, since $x_{\epsilon} \in{\overline{\mathcal{O}_{s_{p-1} \cdots s_{1} \cdot \sigma_{p}}}}^{w k}$, there is $s_{p} \in S$ such that $\widehat{s_{p} \cdots s_{1}}\left(\sigma_{p}\right)-x_{\epsilon} \in V_{\epsilon}^{\prime}$.

Given $n \in \mathbb{N}$, if $x \in \widehat{s_{n} \cdots s_{1}}\left(B\left[\sigma_{n}, \delta_{\epsilon}\right] \cap \Omega^{*}\right)$, let $x:=\widehat{s_{n} \cdots s_{1}}\left(\sigma_{n}+z_{x}\right)$ for some $z_{x} \in B\left[0, \delta_{\epsilon}\right]$. Then by non-expansiveness of the action, we have that

$$
\begin{aligned}
\left\|\widehat{s_{n} \cdots s_{1}}\left(\sigma_{n}\right)-x\right\| & =\left\|\widehat{s_{n} \cdots s_{1}}\left(\sigma_{n}\right)-\widehat{s_{n} \cdots s_{1}}\left(\sigma_{n}+z_{x}\right)\right\| \\
& \leq\left\|\sigma_{n}-\left(\sigma_{n}+z_{x}\right)\right\|=\left\|z_{x}\right\| \\
& \leq \delta_{\epsilon} .
\end{aligned}
$$

This above inequality yields the following inclusions :

$$
\begin{aligned}
\widehat{s_{n} \cdots s_{1}}\left(B\left[\sigma_{n}, \delta_{\epsilon}\right] \cap \Omega^{*}\right) & \subset B\left[\widehat{s_{n} \cdots s_{1}}\left(\sigma_{n}\right), \delta_{\epsilon}\right] \cap \Omega^{*} \\
& \subset x_{\epsilon}+V_{\epsilon}^{\prime}+V_{\epsilon}^{\prime} \\
& \subset x_{\epsilon}+V_{\epsilon} .
\end{aligned}
$$

Hence for all $n \in \mathbb{N}$, we have $\widehat{s_{n} \cdots s_{1}}\left(B\left[\sigma_{n}, \delta_{\epsilon}\right] \cap \Omega^{*}\right) \subset x_{\epsilon}+V_{\epsilon}$. So we have :

$$
\Omega^{*}=\bigcup_{i=1}^{\infty} B\left[\sigma_{i}, \delta_{\epsilon}\right] \cap \Omega^{*} \subset \bigcup_{i=1}^{\infty}{\widehat{s_{i} \cdot s_{1}}}^{-1}\left(x_{\epsilon}+V_{\epsilon}\right)
$$


Then $\left\{{\widehat{s_{i} \cdots s_{1}}}^{-1}\left(x_{\epsilon}+V_{\epsilon}\right) \cap \Omega^{*} ; i=1,2, \cdots\right\}$ is a weak open covering of the weakly compact set $\Omega^{*}$. Therefore there is $m \in \mathbb{N}$ such that $\Omega^{*}=\bigcup_{i=1}^{m} \widehat{s_{i} \cdots s_{1}}-1$ $\left(x_{\epsilon}+V_{\epsilon}\right) \cap \Omega^{*}$. From lemma 2 we know that $\widehat{s_{m} \cdots s_{1}}\left(\Omega^{*}\right)=\Omega^{*}$ therefore it follows

$$
\begin{aligned}
\Omega^{*}=\bigcup_{i=1}^{m}{\widehat{s_{i} \cdots s_{1}}}^{-1}\left(x_{\epsilon}+V_{\epsilon}\right) \cap \Omega^{*} & \subset \bigcup_{i=1}^{m} s_{m} \widehat{\cdots s_{i}+1}\left(\left(x_{\epsilon}+V_{\epsilon}\right) \cap \Omega^{*}\right) \\
& \subset \bigcup_{i=1}^{m} s_{m} \widehat{\cdots s_{i}+1}\left(B\left[x_{\epsilon}, \epsilon\right] \cap \Omega^{*}\right)
\end{aligned}
$$

By non-expansiveness, we have $\left\|s_{m} \widehat{\cdots \cdot s_{i}+1}(y)-s_{m} \widehat{\cdots s_{i+1}}\left(x_{\epsilon}\right)\right\| \leq \epsilon$, for all $y \in B\left[x_{\epsilon}, \epsilon\right]$. Thus we have the following inclusion

$$
\Omega^{*} \subset \bigcup_{i=1}^{m} B\left[s_{m} \widehat{\cdots s_{i+1}}\left(x_{\epsilon}\right), \epsilon\right] .
$$

Therefore $\Omega^{*}$ is norm totally bounded. On the other hand, since it is weakly closed, it is norm complete. Hence, these two facts imply $\Omega^{*}$ is compact in the topology induced by the norm.

Now we are ready to proceed to the proof of Theorem 1.

Proof. By a Zorn's lemma argument we fix a minimal non-void weak ${ }^{*}$ compact convex subset $K^{*}$ of $K$. From lemma 2 , there is a minimal non-empty, weak* compact set $\Omega^{*} \subset K^{*}$ with the property that $s . \Omega^{*}=\Omega^{*}$ for all $s \in S$.

- Step 1: $S$ is a separable or seq-LA semi-topological semigroup.

Then by lemma $4, \Omega^{*}$ is norm compact; and so is its closed convex hull (by Mazur's theorem). If it has a positive diameter then, by [7, lemma 1], there is $u \in \overline{c o}\left(\Omega^{*}\right)$ such that :

$$
r:=\sup _{x \in \Omega^{*}}\|x-u\|<\delta\left(\Omega^{*}\right) \quad(*) .
$$

As readily checked, the set $K_{*}:=\bigcap_{x \in \Omega^{*}} B[x, r] \cap K^{*}$ is a non-void (because contains $u$ ) weak* compact (as a weak ${ }^{*}$ closed subset of $K^{*}$ ), convex (intersection of convex sets), proper (because of $(*))$ subset of $K^{*}$ which is also $S$-invariant. In fact, given $s \in S$ if we fix $x \in \Omega^{*}$ and $y \in K_{*}$ then, $x=$ s.z for some $z \in \Omega^{*}$ (because $\left.s . \Omega^{*}=\Omega^{*}\right)$. Therefore, $\|x-s . y\| \leq\|z-y\| \leq r \Rightarrow s . y \in B[x, r] \cap K^{*}$. Hence s.y $\in K_{*}$. Therefore by minimality of $K^{*}, K_{*}=K^{*}$ which is absurd since $K_{*}$ is a proper subset.

- Step 2: We assume that $S$ is $\sigma$-LA.

Let $S=\cup_{\gamma \in \Gamma} S_{\gamma}$ such that for all $\gamma, \gamma^{\prime} \in \Gamma$, there is $\gamma^{\prime \prime} \in \Gamma$ such that $S_{\gamma} \cup S_{\gamma^{\prime}} \subset S_{\gamma^{\prime \prime}}$ where each $S_{\gamma}$ is separable. Define

$$
\gamma \leq \gamma^{\prime} \text { if and only if } S_{\gamma} \subset S_{\gamma^{\prime}}
$$


By the first step, for all $\gamma \in \Gamma$, the restriction $S_{\gamma} \times K \rightarrow K$ of the $S$-action on $K$ possesses a common fixed point in $K$ which we denote by $x_{\gamma}$. Since $(\Gamma, \leq)$ is a directed set, then $\left(x_{\gamma}\right)_{\gamma \in \Gamma}$ defines a net of elements of $K$ such that $s . x_{\gamma}=x_{\gamma}$ for all

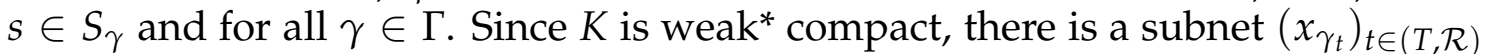
of $\left(x_{\gamma}\right)_{\gamma \in \Gamma}$ which is weak* convergent. Let $\hat{x}:=\mathrm{wk}^{*}-\lim _{t} x_{\gamma_{t}} \in K$ be its weak limit. We shall show that $\hat{x}$ is a common fixed point for $S$ in $K$. Let us fixed $s \in S$. Since $S=\cup_{\gamma \in \Gamma} S_{\gamma}$, let $\gamma_{s} \in \Gamma$ such that $s \in S_{\gamma_{s}}$. Fix $t_{s} \in T$ such that $t_{s} \mathcal{R} t \Rightarrow$ $\gamma_{s} \leq \gamma_{t}$ (this implies that $s \in S_{\gamma_{t}}$ ). Note that such a $t_{s}$ does exist because $\left(S_{\gamma_{t}}\right)_{t}$ is a subnet of $\left(S_{\gamma}\right)_{\gamma}$. On the other hand, by weak* continuity, we have $s . \hat{x}-\hat{x}=\mathrm{wk}^{*}-$ $\lim _{t}$ s. $x_{\gamma_{t}}-x_{\gamma_{t}}$. Since the norm of the dual is weak* lower semi-continuous, given $\epsilon>0$ there is $t_{\epsilon} \in T$ such that $\liminf _{t}\left\|s \cdot x_{\gamma_{t}}-x_{\gamma_{t}}\right\| \leq \inf _{t_{\epsilon} R t}\left\|s \cdot x_{\gamma_{t}}-x_{\gamma_{t}}\right\|+\epsilon$. Now let $t_{\epsilon}^{s} \in T$ such that $t_{\epsilon} \mathcal{R} t_{\epsilon}^{s}$ and $t_{s} \mathcal{R} t_{\epsilon}^{s}$. Then $t_{\epsilon}^{s} \mathcal{R} t \Rightarrow s \in S_{\gamma_{t}} \Rightarrow s \cdot x_{\gamma_{t}}=x_{\gamma_{t}}$. Therefore the following inequalities hold

$$
\begin{aligned}
\|s . \hat{x}-\hat{x}\| & \leq \liminf _{t}\left\|s \cdot x_{\gamma_{t}}-x_{\gamma_{t}}\right\| \\
& \leq \inf _{t_{\epsilon} \mathcal{R} t}\left\|s \cdot x_{\gamma_{t}}-x_{\gamma_{t}}\right\|+\epsilon \\
& \leq \inf _{t_{\epsilon}^{\mathcal{S}} t}\left\|s \cdot x_{\gamma_{t}}-x_{\gamma_{t}}\right\|+\epsilon \\
& =\epsilon
\end{aligned}
$$

Hence, $\|s . \hat{x}-\hat{x}\| \leq \epsilon$ for all $\epsilon>0$ which implies $s \hat{x}=\hat{x}$ for all $s \in S$.

- Step 3: We assume that $S$ is strongly left reversible

Since a strongly left reversible semi-topological semigroup $S$ is a direct union of a family of countable (left reversible) sub-semigroups $S_{\gamma}$, if we consider the restriction of the $S$-action on each $S_{\gamma} \times K$ when $\gamma$ runs through $\Gamma$, and fix a minimal non-empty weak ${ }^{*}$ compact convex and $S_{\gamma}$-invariant subset $K_{\gamma}$ of $K$, then [20] guarantees the existence of a minimal non-void weak ${ }^{*}$ compact subset $\Omega_{\gamma}^{*} \subset K_{\gamma}^{*}$ such that $s . \Omega_{\gamma}^{*}=\Omega_{\gamma}^{*}$ for all $s \in S_{\gamma}$. Then using Step 1, the sub-semigroup $S_{\gamma}$ possesses a common fixed point $x_{\gamma}$ in $K$. By considering the family $\left(x_{\gamma}\right)_{\gamma}$, a similar argument as in Step 2 shows that under a suitable pre-order on $\Gamma$, it becomes a net with a weak* convergent subnet converging to a common fixed point for $S$.

From Theorem 1, we derive the following result which is more easy to handle in applications.

Theorem 2. Let $S$ be a semi-topological semigroup satisfying the conditions of Theorem 1 . Then it has the following property:

$\left(F_{\text {weak }}^{*}\right)$ : Whenever $S \times K \rightarrow K$ is a weak ${ }^{*}$ jointly continuous non-expansive action on a non-empty weak* compact convex subset $K$ of a dual $E^{*}$ of a Banach space $E$, such that for all non-void weak* closed and $S$-invariant subset $B$ of $K$ with the property $s . B=B$ for all $s \in S$, there is $x \in B$ whose orbit $\mathcal{O}_{x}$ is relatively compact in the weak topology; then there is in $K$ a common fixed point for $S$.

Proof. Indeed, the weak topology $\sigma\left(E^{*}, E^{* *}\right)$ is finer than the locally convex topology generated by $\operatorname{Ext}\left(B_{E^{* *}}\right)$. 
We know that discrete left amenable semigroups and separable left amenable semi-topological semigroups have the above fixed point property. A natural question to raise at this point is the following :

Question 1 : Do left amenable semi-topological semigroups possess the fixed point property $\left(F_{\text {weak }}^{*}\right)$ ?

Remark 1. We point out that, theorem 1 is related to the following long-standing and difficult question raised by A. T. -M. Lau in 1976 which can be stated as follows :

Does any left amenable semi-topological semigroup $S$ possess the following fixed point property :

$\left(F^{*}\right)$ : Whenever $S$ defines a jointly weak ${ }^{*}$ continuous non-expansive action on a non-void, weak* compact convex set $K$ in a dual $E^{*}$ of a Banach space $E$, then $S$ has a common fixed in $K$.

The converse of this question is true, just by looking at the action of the adjoints of left translation operators on the set of all means on LUC (which is a non-void weak $^{*}$ compact convex set). If the answer to this question is affirmative, then $\left(F^{*}\right)$ will be a non-linear fixed point characterization of left amenable semi-topological semigroups. While an affine characterization was established by Mitchell [24].

Remark 2. In our best knowledge up to now, the answer of this question is affirmative for commutative semigroups [3]. Unfortunately, the proof for the commutative case does not use the fact that such semigroups are left amenable, but strongly the abelian property. So we still do not know for the general case, whether the answer is positive. On the other hand, our work shows that with an additional assumption on $K$, it is possible to guarantee the non-emptiness of the fixed point set. For instance, that is the case if $K$ has normal structure [20]; or norm separable [21]. These partial results show that, the answer to this question is very related to the geometrical and topological structures of $K$. Up to now, some partial noncommutative answers have been established (see [18],[20],[21]).

Theorem 3. Let $S$ be a semi-topological semigroup. If $\operatorname{LUC}(S)$ has a $\mathrm{LIM}$, then $S$ has the following fixed point property :

$\left(F_{i s o m}^{*}\right)$ : Whenever $S \times K \rightarrow K$ is a jointly weak ${ }^{*}$ continuous non-expansive action of $S$ on a non-empty weak* compact convex subset $K$ of a dual Banach space $E^{*}$ such that there is a weak ${ }^{*}$ closed isometry from $K$ into $\ell^{1}$, then there is in $K$ a common fixed point for $S$.

Remark 3. In the above theorem, we consider $\ell^{1}$ as the dual of $c_{0}$ and point out that the isometry in the above theorem need not be linear, but only closed in the weak ${ }^{*}$ topology (i.e., the direct image of a weak* closed subset of $K$ is weak ${ }^{*}$ closed in $\left.\ell^{1}\right)$. 
Proof. By [28, lemma 2.12], there is a non-void weak* compact subset $\omega_{w k *}$ of $K$ with the property :

$$
\text { s. } \omega_{w k *}=\omega_{w k *} \text { for all } s \in S .
$$

Let $\phi: K \rightarrow \ell^{1}$ be the isometry whose existence is guaranteed by assumption. Then by [49], the set

$$
C:=\left\{x \in \phi(K) ; \sup _{y \in \phi\left(\omega_{w k *}\right)}\|x-y\|=r\right\}
$$

where $r:=\inf _{y \in \phi(K)} \sup _{z \in \phi\left(\omega_{z w k *}\right)}\|y-z\|$, is a non-void and norm compact set. Therefore its preimage $\phi^{-1}(C)$ is a non-empty norm compact subset of $E^{*}$. Now define analogously as $C$ the set

$$
\hat{K}:=\left\{x \in K ; \sup _{y \in \omega_{w k *}}\|x-y\|=\rho\right\} .
$$

where $\rho:=\inf _{y \in K} \sup _{z \in \omega_{w k *}}\|y-z\|$. The set $\hat{K}$ is non-void because on the one hand, it can be written as

$$
\bigcap_{j}\left\{x \in K ; \sup _{y \in \omega_{w k *}}\|x-y\| \leq \rho+\frac{1}{j}\right\}
$$

and on the other hand, each set in the intersection is non-empty and weak ${ }^{*}$ closed due to the weak ${ }^{*}$ lower-semi-continuity of the dual norm on $E^{*}$. Hence, the weak compactness of $K$ forces $\hat{K}$ to be non-void. Next we show that $\hat{K}$ is norm compact. For that, it is enough to prove that it is a subset of $\phi^{-1}(C)$. Fortunately, it does. In fact, given $x \in \hat{K}$ we have $\phi(x) \in \phi(K)$ and

$$
\begin{aligned}
\sup _{y \in \phi\left(\omega_{w k *}\right)}\|\phi(x)-y\| & =\sup _{y \in \omega_{w k *}}\|\phi(x)-\phi(y)\| \\
& =\sup _{y \in \omega_{w k *}}\|x-y\|=\rho \\
& =\inf _{y \in K} \sup _{z \in \omega_{w k *}}\|y-z\| \\
& =\inf _{y \in K} \sup _{z \in \omega_{w k *}}\|\phi(y)-\phi(z)\| \\
& =\inf _{y \in \phi(K)} \sup _{z \in \phi\left(\omega_{w k *}\right)}\|y-z\|=r
\end{aligned}
$$

Therefore the inclusion holds and it follows that $\hat{K}$ is a non-empty norm compact convex subset of $K$. We prove that $\hat{K}$ is $S$-invariant. Let $x \in \hat{K}$ and $s \in S$ fixed. Given $y \in \omega_{w k *}$, using the property $\left.{ }^{*}\right)$ we let $y=s . z$ for some $z \in \omega_{w k *}$. Then using the non-expansiveness of the action, we get :

$$
\begin{aligned}
\|s . x-y\| & =\|s . x-s . z\| \\
& \leq\|x-z\| \\
& \leq \sup _{y \in \omega_{w k * *}}\|x-y\|=\rho
\end{aligned}
$$


Therefore it follows that

$$
\sup _{y \in \omega_{w k *}}\|s . x-y\|=\rho
$$

Thus, s. $x \in \hat{K}$; i.e., $\hat{K}$ is invariant under $S$. It follows that the restriction $S \times \hat{K} \rightarrow \hat{K}$ of the $S$-action on $\hat{K}$ is jointly continuous in the norm topology (since norm and weak* topologies agree on $\hat{K}$ ). Using a Zorn's lemma argument if necessary, we may assume that $\hat{K}$ is minimal (i.e., there is no proper subset of $\hat{K}$ which is nonvoid, norm compact convex and $S$-invariant). Then $\hat{K}$ must be a singleton because otherwise, being norm compact and convex, it has normal structure (see [7]) and therefore a similar argument as in Step 1 in the proof of Theorem 1 leads us to a contradiction.

The following question appears natural from this result.

Question 2 : Can we remove or weaken the weak* closeness assumption of the isometry in Theorem 3 ?

Corollary 1. Any $\sigma$-LA semi-topological semigroup $S$ possesses the fixed point property $\left(F_{i s o m}^{*}\right)$.

Proof. In fact, this follows using a similar argument as in Step 2 in the proof of Theorem 1.

When $S$ is left reversible as a semi-topological semigroup, then (see [12] or [21]), $S$ becomes a directed set if we let :

$$
a \leq b \text { iff }\{b\} \cup \overline{b . S} \subset\{a\} \cup \overline{a . S}
$$

Then if fix $x \in K$ (i.e., whenever $S$ defines an action as in theorem 3) we define $\Omega_{S}:=\overline{s . S . x}$ for all $s \in S$. We obtain a decreasing net of non-void subsets of $K$ whose asymptotic center $\mathrm{AC}\left(\left(\Omega_{S}\right)_{s \in S} ; K\right)$ is non-void, norm compact, convex and $S$-invariant. Hence, it follows:

Corollary 2. All left reversible semi-topological semigroups possess the fixed point property $\left(F_{i s o m}^{*}\right)$.

\subsection{A non-linear common fixed point property in Banach spaces}

Using Theorem 1, we derive the following result which is a dual version of the fixed point property $F_{\tau}^{*}$.

Theorem 4. Let $S$ be a semi-topological semigroup. Assume that it satisfies either one of the following conditions :

1. $S$ is $\sigma$-LA;

2. $S$ is seq-LA;

3. $S$ is strongly left reversible.

Then $S$ possesses the following fixed point property :

$\left(F_{\text {weak }}\right)$ : Whenever $S \times K \rightarrow K$ is a jointly weakly continuous non-expansive action of $S$ on a non-empty weakly compact convex subset $K$ of a Banach space $E$, then there is in $K$ a common fixed point for $S$. 
Proof.

- Step 1 : We first assume that $S$ is separable or seq-LA. We embed $E$ in its second dual $E^{* *}$ through the canonical injection $j: E \rightarrow E^{* *}$ which is an isomorphism from $(E, \mathrm{wk})$ onto $\left(j(E), \mathrm{wk}^{*}\right)$. Then $\hat{K}:=j(K)$ is a non-void weak ${ }^{*}$ compact convex subset of $E^{* *}$. We carry the $S$-action on $K$ to $\hat{K}$ by letting $s * j(x):=j(s . x)$, for all $s \in S$ and $x \in K$. As readily checked, the action $S \times \hat{K} \rightarrow \hat{K}$ is jointly weak* continuous and norm non-expansive. Let $\hat{\tau}$ be the locally convex topology on $E^{* *}$ induced by the extreme points of $B_{E^{* * *}}[0,1]$ (the unit closed ball of the dual of $\left.E^{* *}\right)$. If $\hat{B} \subset \hat{K}$ is a non-void weak* compact subset such that $s * \hat{B}=\hat{B}$ for all $s \in S$, using a Zorn's lemma argument if necessary together with [28, lemma 2.12] if $S$ is left amenable or [20, corollary 3.7] if $S$ is left reversible, we may assume that $\hat{B}$ is minimal (in the sense that, if $\widetilde{B}$ is a non-void weak* compact $S$-invariant set contained in $\hat{B}$, then $\tilde{B}=\hat{B})$. Then $B:=j^{-1}(\hat{B})$ is a minimal non-empty weakly compact $S$-invariant and separable subset of $K$ with the property that $s . B=B$ for all $s \in S$. Therefore using lemma 4 , it follows that $B$ is norm compact and a fortiori its image $\hat{B}$. Thus, for all $j(x) \in \hat{K}$, the orbit $\mathcal{O}_{j(x)}$ is relatively $\hat{\tau}$-compact (since norm and $\hat{\tau}$ topologies agree on the norm closed orbit). Hence by Theorem 1 , there is $\hat{x} \in K$ such that $s * j(\hat{x})=j(\hat{x})$ for all $s \in S$. Hence, $\hat{x}$ is a common fixed point for $S$ due to the injectivity of $j$.

- Step 2 : Now we assume that $S$ is an arbitrary semi-topological semigroup with either one of the properties in the theorem. From Step 1 , a similar argument as in the proof of theorem 1, shows that if we consider the action of $S$ carried on $E^{* *}$, then we have

$$
F(S):=\{x \in K ; s * j(x)=j(x) \text { for all } s \in S\} \neq \varnothing .
$$

Hence using the injectivity of $j$, the non-empty set $F(S)$ is contained in the fixed point set of $S$; which proves the existence of a common fixed point.

Remark 3. Theorem 4 extends Hsu [13, theorem 4], because discrete left reversible semigroups are strongly left reversible (nice proof due to Hsu [13, lemma 1]) and Mitchell [26]. On the other hand, it shows that joint weak continuity condition is a sufficient condition for avoiding the use of normal structure when dealing with non-expansive actions on weak compact convex sets.

Discrete left reversible semigroups and separable left reversible semi-topological semigroups possess the previous fixed point property. However, we may ask:

Question 3 : Do left reversible semi-topological semigroups possess the fixed point property $\left(F_{\text {weak }}\right)$ ?

Since a left amenable semi-topological semigroup $S$ need not be $\sigma$-LA, the following question is natural :

Question 4: Do left amenable semi-topological semigroups have the fixed point property $\left(F_{\text {weak }}\right)$ ?

Acknowledgment This paper is partly contained in the $\mathrm{PhD}$ thesis of the author under the supervision of Professor Anthony To-Ming Lau. 


\section{References}

[1] D. Alspach, A fixed point free nonexpansive map, Proc. Amer. Math. Soc. (1981), 423-424.

[2] J. F. Berglund, H. D. Junghenn and P. Milnes, Analysis on Semigroups, John Wiley \& Sons, New York, (1989).

[3] S. Borzdynski and A. Wisnicki, A common fixed point theorem for commuting family of weak* continuous non-expansive mappings, Studia Math. 225 (2014), 173-181.

[4] M. S. Brodskii and D. P. Milman, On the center of a convex set, Dokl. Akad. Nauk SSSR 59 (1948), 837-840.

[5] M. M. Day, Amenable semigroups, Illinois J. Math. 1 (1957), 509-544.

[6] M. M. Day, Means for the bounded functions and ergodicity of the bounded representations of semigroups, Trans. Amer. Math. Soc., volume 69, (1950), 276-291.

[7] R. DeMarr, Common fixed points for commuting contraction mappings. Pacific J. Math. 13 (1963), 1139-1141.

[8] E. Granirer, On amenable semigroups with a finite dimensional set of invariant means II, Illinois J. Math. (1963), 49-58.

[9] E. Granirer, On invariant mean on topological semigroups and on topological groups Pacific J. Math., 15 (1965), 107-140.

[10] E. Hewitt, On two problems of Urysohn, Ann. of Math, 47 (1946), 503-509.

[11] E. Hewitt and Kenneth A. Ross, Abstract Harmonic Analysis volume 1.

[12] R. D. Holmes and A. T. -M. Lau, Non-expansive actions of topological semigroups and fixed points, J. London Math. Soc. (2), 5 (1972), 330-336.

[13] R. Hsu, Topics on weakly almost periodic functions, PhD thesis, SUNY at Buffalo, 1985.

[14] G. Itzkowitz and T. Mitchell, Topological semigroups and fixed points II, Notices Amer. Math. Soc., volume 13, (1966), 725.

[15] L. A. Karlovitz, On nonexpansive mappings, Proc. Amer. Math. Soc., 55 (1976), 321-325.

[16] W. A. Kirk, A fixed point theorem for mappings which do not increase distances, Amer. Math. Monthly, Volume 72, (1965), 1004-1006.

[17] A. T. -M. Lau, Invariant means on almost periodic functions and fixed point properties, Rocky Mountain J. Math, volume 3, (1973), 69-76. 
[18] A. T. -M. Lau, Amenability and fixed point property for semigroup of nonexpansive mappings, in : M.A. Thera, J.B. Baillon(Eds.), Fixed Point theory and Applications, in: PitmanRes. Notes Math. Ser., volume 252, Longman Sci. Tech., Harlow, (1991), pp. 303-313.

[19] A. T. -M. Lau and Y. Zhang, Fixed point properties of semigroups of nonexpansive mappings, J. Funct. Anal. 254, (2008), 2534-2554.

[20] A. T. -M. Lau and Y. Zhang, Fixed point properties for semigroups of nonlinear mappings and amenability, Journal of Functional Analysis, Volume 263, (2012), 2949-2977.

[21] A. T. -M. Lau and W. Takahashi, Invariant means and fixed point properties for non-expansive representations of topological semigroups, Topological Methods in Nolinear Analysis Journal of the Juliusz Schauder Center, Volume 5, (1995), 39-57.

[22] T. C. Lim, Characterizations of normal structure, Proceedings of the AMS, volume 43, (1974), 313-319.

[23] T. C. Lim, Asymptotic centers and nonexpansive mappings in conjugate Banach spaces, Pacific Journal of Math, volume 90,(1980), 135-143.

[24] T. Mitchell, Topological semigroups and fixed points, Illinois J. Math. 14, (1970), 630-641.

[25] T. Mitchell, Fixed point of reversible semigroups of non-expansive mappings, Kodai Math. Seminar Rep. 22, (1970), 322-323.

[26] W. B. Moors, A characterization of weak compactness in Banach spaces, Bull. Austral. Math. Soc. Volume 55, (1997), 497-501.

[27] N. Randrianantoanina, Fixed point properties of semigroups of nonexpansive mappings, Journal of Functional Analysis, 258, (2010), 3801-3817.

[28] K. Salame, Nonlinear common fixed point properties for semi-topological semigroups in uniformly convex spaces (Accepted, Journal of Fixed Point Theory and Applications).

[29] W. Takahashi , Fixed point theorem for amenable semigroups of nonexpansive mappings, Kodai Math. Rep. 21 (1969), 383-386.

Department of Mathematical and Statistical Sciences

University of Alberta, Edmonton, Alberta T6G 2G1, Canada

E-mail address : khadime@ualberta.ca 